\title{
EDIFİCATE
}

I Congreso de Escuelas de Edificación y Arquitectura Técnica de España València, 4 y 5 de noviembre de 2021

Escuela Técnica Superior de Ingeniería de Edificación

Universitat Politècnica de València

Doi:https://doi.org/10.4995/EDIFICATE2021.2021.13497

\section{Estrategias docentes en el proceso de enseñanza y aprendizaje sobre sostenibilidad en el Grado en Arquitectura Técnica de la Universidad de Burgos}

\section{Strategies in teaching and learning process on sustainability in the Degree in Technical Architecture of the University of Burgos}

\author{
Javier Garabito López ${ }^{\mathrm{a}}$, Ángel Rodríguez Saiz ${ }^{\mathrm{b}}$, Verónica Calderón Carpinteroc, Sara \\ Gutiérrez González $^{\mathrm{d}}$, Francisco Fiol Oliváne y Carmelo Muñoz Ruipérez ${ }^{\mathrm{f}}$ \\ Departamento de Construcciones Arquitectónicas e Ingeniería de la Construcción y del Terreno, \\ Escuela Politécnica Superior de la Universidad de Burgos \\ a jgarabito@ubu.es, barsaizmc@ubu.es, cvcalderon@ubu.es, ${ }^{\mathrm{d}}$ sggonzalez@ubu.es, effiol@ubu.es y \\ 'cmruip@ubu.es
}

\begin{abstract}
The University of Burgos faced the 2010-2011 academic year the challenge of starting a new Degree in Technical Architecture, which was the adaptation of the technical architecture studies in Spain according to Bologna System. One of the goals was to introduce sustainability criteria in this academic qualification, so new subjects were taught: Sustainable Construction and Energy Efficiency. As a novelty the programs of these subjects include such important topics as: Life Cycle Assessment, Sustainability Assessment Tools, sustainability criteria... This new approach to the studies has not limited only to the new subjects. Materials Fundamentals, as an example, raise new issues, such as waste management or recycling. A methodology used in Sustainable Construction is the cooperative teaching-learning method, through the critical study of existing sustainable buildings to later transform a conventional building into a sustainable one.
\end{abstract}

Keywords: Teaching methodology, skills, cooperative work, sustainability, Sustainable Construction, Materials

\section{Resumen}

La Universidad de Burgos afrontó el curso 2010-2011 el reto de iniciar un nuevo Grado en Arquitectura Técnica, que consistía en la adecuación de los estudios de Arquitectura Técnica en España según el Sistema de Bolonia. Uno de los objetivos era introducir criterios de sostenibilidad en esta titulación académica, por lo que se impartieron nuevas materias: Construcción Sostenible y 
Estrategias docentes en el proceso de enseñanza y aprendizaje sobre sostenibilidad en el Grado en Arquitectura Técnica de la Universidad de Burgos - Strategies in teaching and learning process on sustainability in the Degree in Technical Architecture of the University of Burgos

Eficiencia Energética. Como novedad los programas de estas asignaturas incluyen temas tan importantes como: Evaluación del Ciclo de Vida, Herramientas de Evaluación de la Sostenibilidad, criterios de sostenibilidad ... Este nuevo enfoque de los estudios no se ha limitado solo a las nuevas asignaturas. Fundamentos de los Materiales, por ejemplo, plantea nuevos contenidos, como la gestión de residuos o el reciclaje. En la asignatura de Construcción Sostenible como metodología se emplea el método de enseñanza-aprendizaje cooperativo, mediante el estudio crítico de edificios sostenibles existentes para, posteriormente, transformar un edificio convencional en uno sostenible.

Palabras clave: Metodología docente, competencias, trabajo cooperativo, sostenibilidad, Construcción Sostenible, Materiales. 


\section{Implantación del concepto de sostenibilidad en el Grado en Arquitectura Técnica.}

\subsection{Antecedentes}

Los estudios de Arquitectura Técnica de la Universidad de Burgos tienen una larga tradición universitaria, ya que la escuela es una de las más antiguas de España. Los estudios de Aparejadores se iniciaron en Burgos el curso académico 1962-1963, dependiendo de la Escuela Técnica Superior de Arquitectura de Madrid.

El 26 de mayo de 1994 se crea la Universidad de Burgos, mediante la Ley 12/94. De este modo, estos estudios universitarios pertenecen a la Universidad de Burgos desde ese año.

La Escuela Politécnica Superior de la Universidad de Burgos se crea en 1998 (RD 210/98 BOCy L. 6 oct. 6) como transformación de la Escuela Politécnica.

El 19 de junio de 1999, 29 ministros de educación europeos firmaron la Declaración de Bolonia, que da el nombre al proceso y en el que se basan los fundamentos del Espacio Europeo de Educación Superior (EEES).

En el año 2004 la Agencia Nacional de Evaluación de la Calidad y Acreditación, ANECA, crea el Libro Blanco del título universitario de Grado en Ingeniería de Edificación - Grsdo en Arquitectura Técnica, estableciendo una estructura de primer nivel o Grado de cuatro cursos lectivos anuales de 240 ECTS.

\subsection{El Grado en Arquitectura Técnica.}

Con el proceso de convergencia europea se propuso adaptar la titulación al Grado en Arquitectura Técnica, siguiendo las directrices de Bolonia. En la Universidad de Burgos aprovechamos la necesidad de la acreditación para renovar y actualizar los programas con el fin de adaptarlos a las necesidades del mundo profesional, más competitivo y en constante cambio.

Entre las nuevas competencias que se recogen en la memoria para la verificación del título podemos destacar:

- Gestionar las nuevas tecnologías edificatorias y participar en los procesos de gestión de la calidad en la edificación.

- Realizar análisis, evaluaciones y certificaciones de eficiencia energética, así como estudios de sostenibilidad en los edificios.

- Dirigir y gestionar el uso, conservación y mantenimiento de los edificios, redactando los documentos técnicos necesarios;

- Elaborar estudios del ciclo de vida útil de los materiales, sistemas constructivos y edificios.

- Gestionar el tratamiento de los residuos de demolición y de la construcción. 
Y como nuevas Competencias Específicas:

- Capacidad para el análisis del ciclo de vida útil de los elementos y sistemas constructivos.

- Conocimiento de la evaluación del impacto ambiental de los procesos de edificación y demolición, sostenibilidad en edificación y de los procedimientos y técnicas para evaluar la eficiencia energética de los edificios.

- Procedimientos y técnicas para evaluar la eficiencia energética de los edificios.

- Conocimiento y aplicaciones de las Energías Renovables aplicadas a la Edificación que se establecen en el artículo 15 de la Parte I del Código Técnico de la Edificación y de otras fuentes de Energías Renovables innovadoras aplicadas a la Edificación.

Así, uno de los objetivos de la adaptación de la titulación fue introducir criterios de sostenibilidad (Edwards, 2008) para el Grado, de modo que se ofertaron dos nuevas asignaturas: Construcción Sostenible y Eficiencia Energética.

La introducción de la Asignatura de Eficiencia Energética, de 3 créditos ECTS, en el Plan formativo de la Titulación del Grado en Arquitectura Técnica, ha supuesto un cambio cualitativo en la formación del profesional Arquitecto Técnico, dotándole de conocimiento relativo a los aspectos de demanda energética de los edificios y capacidad de decisión para aportar soluciones que fomenten el ahorro energético a través de soluciones de rentabilidad energética, introduciendo criterios de arquitectura bioclimática en las fases de diseño, ejecución y uso de los edificios.

La asignatura de Construcción Sostenible se analiza en el apartado 2.

Este nuevo enfoque en la titulación no se ha limitado a estas nuevas asignaturas, ya que las asignaturas tradicionales desarrollan competencias directamente relacionadas con la sostenibilidad. Las disciplinas de materiales de construcción consideran la sostenibilidad ambiental como un valor a fomentar.

El curso Fundamentos de los Materiales, figura 1, ha incorporado la Unidad Docente Impacto Ambiental, Gestión y Residuos de los Materiales de Construcción. Su ubicación como última Unidad Docente en la asignatura responde a que los alumnos ya tienen una noción general del concepto de material de construcción y esta Unidad aporta conocimiento sobre aspectos de sostenibilidad que después podrán aplicar en materiales más avanzados estudiados en asignaturas de cursos superiores. 


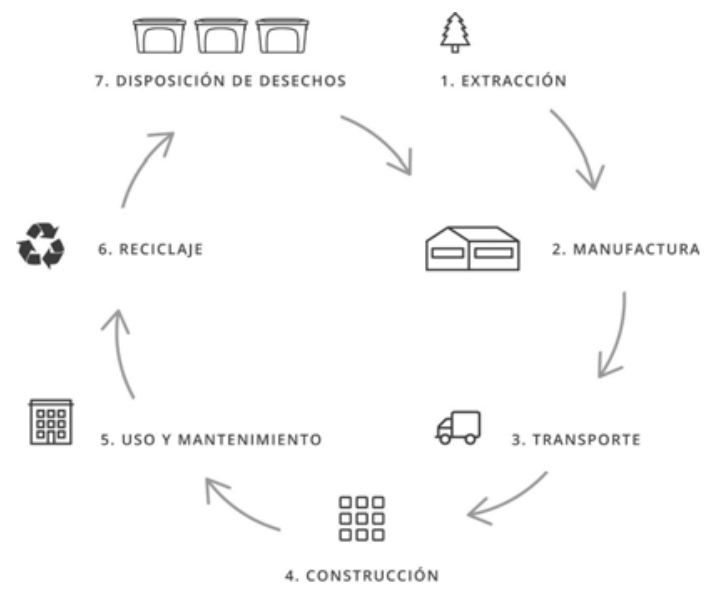

Fig. 1 Fundamentos de Materiales, un ejemplo de presentación

El sector de la Edificación en Europa y en España es responsable de gran parte de la contaminación atmosférica y de aproximadamente el $25 \%$ - $30 \%$ de los residuos de la industria en general. La construcción de los edificios comporta unos impactos ambientales por el uso de materiales que provienen de recursos naturales, la utilización de grandes cantidades de energía tanto en la construcción como a lo largo de su vida y el impacto ocasionado en el emplazamiento. El material, fuertemente manipulado, utilizado en el campo de la construcción tiene unos efectos medioambientales muy importantes, con un gasto muy elevado de energía. No se pueden olvidar los costes ecológicos que suponen tanto la extracción de los recursos minerales (canteras, minas, etc.) como la deposición de los residuos originados, en la construcción o en el derribo, que abarcan desde las emisiones tóxicas al envenenamiento de las aguas subterráneas por parte de los vertedores.

El reciclaje y la reutilización de los residuos de demolición y de la construcción es una solución que mitigará el importante impacto ambiental que genera el vertido y la incineración. La aplicación de los criterios de sostenibilidad y de una utilización racional de los recursos naturales disponibles en la construcción requerirá realizar unos cambios importantes en los valores que ésta tiene como cultura propia. Estos criterios o, más correctamente, principios de sostenibilidad llevarán hacia una conservación de los recursos naturales, una maximización en la reutilización de los recursos, una gestión del ciclo de vida, así como reducciones de la energía utilizada.

\section{La asignatura de Construcción Sostenible}

La Asignatura de Construcción Sostenible, de 3 créditos ECTS, aparece por primera vez en los estudios del Grado en Arquitectura Técnica de la Universidad de Burgos con la adaptación del Plan de Estudios a Bolonia. 
En el programa de Construcción Sostenible se enseñaron por primera vez en Arquitectura Técnica conceptos tan relevantes como: análisis del ciclo de vida, herramientas para evaluar la sostenibilidad como LEED, BREEAM o VERDE, criterios de sostenibilidad, ...

Las competencias específicas que deben adquirir los alumnos en esta asignatura son:

- ETE.04 - Conocimiento de la normativa técnica al proceso de edificación

- ETE.08 - Capacidad para generar documentos de especificación técnica de los procedimientos y métodos constructivos de edificios. Plantear y resolver soluciones constructivas

- $\quad$ ETE.10 - Conocimiento de la evaluación del impacto ambiental de los procesos de edificación y demolición, sostenibilidad en edificación y de los procedimientos y técnicas para evaluar la eficiencia energética de los edificios.

Esta asignatura aporta a los alumnos la sensibilidad por los nuevos valores sociales de la construcción, como el urbanismo respetuoso con el entorno, la utilización de nuevas tecnologías energéticas, la incorporación de nuevos métodos constructivos, el uso de materiales sostenibles, mantenimiento y la rehabilitación de edificios, entre otros. De este modo con el nuevo Plan de Estudios la Universidad de Burgos se incorpora a la vanguardia de la formación universitaria de Técnicos Especialistas en Construcción y Edificación capaces de adaptarse a la evolución social y técnica de nuestra sociedad.

Es una asignatura muy relevante para incentivar la creatividad de los alumnos, ya que el concepto de sostenibilidad es un valor social unido al progreso científico y tecnológico, con constantes avances técnicos de aplicación a la arquitectura, la ingeniería y la construcción.

Como objetico básico, la metodología de trabajo seguida en la asignatura ha tratado de motivar a los alumnos a establecer criterios sobre la aplicación de materiales de construcción respetuosos con el Medio Ambiente, la utilización de instalaciones fáciles de mantener y con un consumo razonable de energía y la configuración de nuevos diseños y soluciones constructivas, más ventajosas para la sostenibilidad y cuidadosas con el medio natural. Por ello, se incentiva el conocimiento de sistemas y procedimientos constructivos sostenibles, energías renovables no contaminantes y materiales reciclados a partir de residuos valorizados, todo ello orientado a la economía circular.

\subsection{Planteamiento de la asignatura de Construcción Sostenible.}

La asignatura, obligatoria, se imparte en el $4^{\circ}$ Curso del Grado en la Universidad de Burgos. A la hora de plantear la asignatura se tuvo en consideración lo siguiente:

- Es una asignatura del Plan de Bolonia, lo que implicaba que la evaluación fuese continua y que el alumno adquiriese competencias específicas y transversales. 
- $\quad$ Aunque los egresados en el Grado en Arquitectura Técnica tienen unas atribuciones limitadas en urbanismo y diseño arquitectónico no se consideraba correcto limitar el contenido a la construcción, ya que si la arquitectura no es sostenible o el urbanismo no plantea criterios de sostenibilidad es prácticamente imposible alcanzar un estándar de calidad.

- Dado que la industria de la construcción está en permanente evolución y se está adaptando a criterios de sostenibilidad, o al menos determinados productos quieren vender esta etiqueta, se consideraba pertinente que el objetivo de las clases teóricas fuese dotar a los alumnos de criterios y conocimientos para que en su labor profesional pudiesen determinar en cada circunstancia qué materiales o procesos constructivos son los más sostenibles y adecuados, mientras que en las clases prácticas los alumnos tendrían que emplear sus conocimientos constructivos en supuestos reales para obtener edificios sostenibles, eficientes y confortables.

- Como metodología educativa consideramos emplear el trabajo cooperativo en los trabajos prácticos, ya que es muy apropiada para desarrollar las habilidades personales de los alumnos, al mismo tiempo que se fomenta la colaboración interactiva con otros compañeros en la realización de proyectos y trabajos conjuntos.

De este modo la asignatura se estructura en dos grandes bloques temáticos formativos complementarios:

- Formación Teórica en conceptos relacionados con la sostenibilidad en construcción, tanto desde el punto de vista urbanístico, de diseño, de procedimientos y técnicas constructivas, así como el empleo de energías renovables y sistemas pasivos.

- Formación Práctica, mediante el desarrollo de dos trabajos específicos de grupo evaluables y con actuación profesional como técnicos expertos, que sirven para poner en valor los conocimientos adquiridos en la formación teórica.

\subsection{Contenidos de la asignatura de Construcción Sostenible.}

El programa de la asignatura está dividido en tres bloques.

En el primer bloque planteamos qué es sostenibilidad y por qué debemos aplicar los criterios de sostenibilidad en la construcción. También definimos cuales pueden ser los criterios de una construcción sostenible. Así mismo se define cual es el marco legislativo y normativo en España. Es un bloque de concienciación del alumno en temas sostenibilidad.

En el segundo bloque explicamos conceptos de urbanismo sostenible, haciendo hincapié en las consecuencias que tiene la urbanización sobre el territorio, desarrollando conceptos como la huella ecológica. También se definen principios del urbanismo sostenible.

El tercer bloque es el más extenso. Este apartado trata sobre arquitectura y construcción sostenible (Hernandez Pezzi, 2007, Reyes et al, 2007). Estudiamos en qué consiste la 
arquitectura sostenible, la importancia de analizar el entorno de las edificaciones, los sistemas pasivos en condiciones de verano e invierno, el análisis de ciclo de vida de materiales o sistemas constructivos. En este bloque finalmente exponemos diferentes sistemas de certificación de la sostenibilidad: LEED, BREEAM y VERDE.

\subsubsection{Formación práctica}

Paralelamente a las clases de teoría, los profesores de la asignatura plantean dos prácticas para su desarrollo en grupo, con no más de tres alumnos. Uno de los objetivos del proceso de enseñanza y aprendizaje es apostar por fomentar el espíritu crítico de los alumnos y crear espacios de diálogo como forma de adquirir conocimientos (De la Torre y Violant, 2001).

Por ello, en la asignatura se ha apostado por utilizar el Estudio de Proyectos de Edificación que han logrado unos niveles relevantes de sostenibilidad, como puede ser una Certificación Sostenible, utilizando los indicadores de calidad de las diferentes entidades de acreditación ambiental. De esta forma, los alumnos se familiarizan con los procedimientos que miden y evalúan el grado de sostenibilidad de un entorno urbanístico o de edificio, así como analizan las carencias y proponen mejoras con criterios de sostenibilidad.

Por otra parte, el segundo trabajo consiste en estudiar y aplicar criterios de sostenibilidad en proyectos de edificación convencionales, es decir, que cumplen estrictamente la normativa. Consideramos que es un excelente modo de enfrentar al alumno al reto de proponer mejoras para alcanzar unos estándares de sostenibilidad en la construcción que logren edificios más confortables, saludables y seguros para sus usuarios.

De este modo, en la asignatura se propone el desarrollo de dos trabajos sobre construcción sostenible, con planteamientos metodológicos totalmente distintos:

1. Estudio de un Proyecto de Edificación Construido con técnicas de sostenibilidad, que dispongan de alguna de Certificación Medioambiental (LEED, BREEAM, HQE, DGNB, VERDE) o un reconocimiento público. Figura 2. 


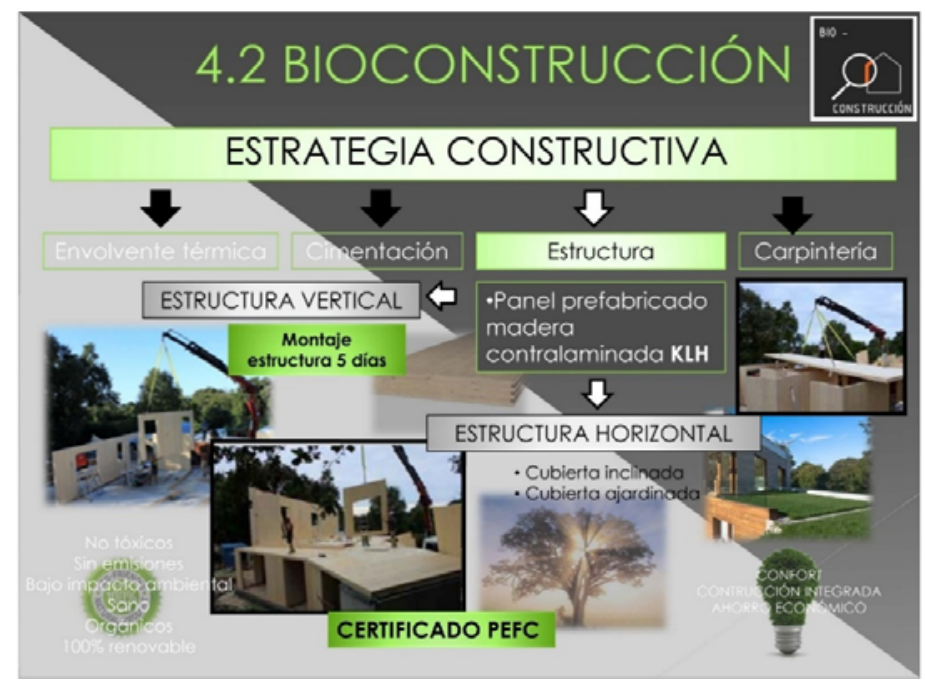

Fig. 2 Construcción Sostenible. Ejemplo de exposición de trabajo práctico 1

2. Desarrollo de un Proyecto de Edificación Sostenible, figura 3: El trabajo consiste en transformar un proyecto de edificación convencional en uno sostenible, aplicando los conocimientos adquiridos en la asignatura (Heywood, 2017). Los alumnos tienen libertad para actuar con un criterio personal, justificando y documentando todos los cambios y razonando por qué han adoptado las soluciones que proponen.

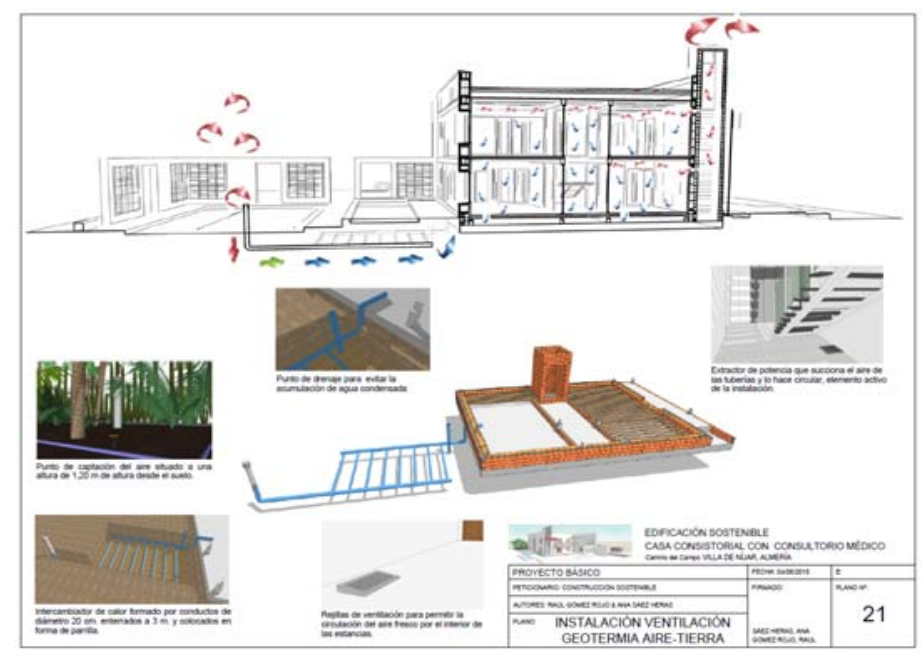

Fig. 3 Construcción Sostenible. Ejemplo de trabajo práctico 2 


\subsubsection{El trabajo cooperativo}

En el nuevo contexto mundial, el éxito de las empresas se basa en configurar equipos de trabajo eficaces, en los que sus miembros puedan aportar sus conocimientos y capacidades de forma complementaria, adaptándose a los requerimientos y necesidades cada vez más flexibles e inciertas del mercado de trabajo. El trabajo en equipo supone una ventaja competitiva sobre los sistemas de organización individuales, menos eficaces y carentes de una visión de conjunto en las estrategias y objetivos empresariales.

Trabajar en grupo no es fácil y requiere el aprendizaje de habilidades y competencias específicas, por lo que desde la Universidad se debe ser sensible a esta nueva realidad social, para formaralumnos bienpreparados y con una alta competencia profesional, pero también personas capaces de crear espacios de interacción personal para trabajar en grupo, compartiendo el conocimiento y comprometidos en conseguir alcanzar el éxito en la gestión de objetivos comunes (Saiz y Gómez, 2011; Marín et. al., 2014).

El trabajo cooperativo en grupo es una metodología educativa apropiada para desarrollar las habilidades personales de los alumnos, al mismo tiempo que se fomenta la colaboración interactiva con otros compañeros para realizar proyectos y trabajos conjuntos. Figura 4.

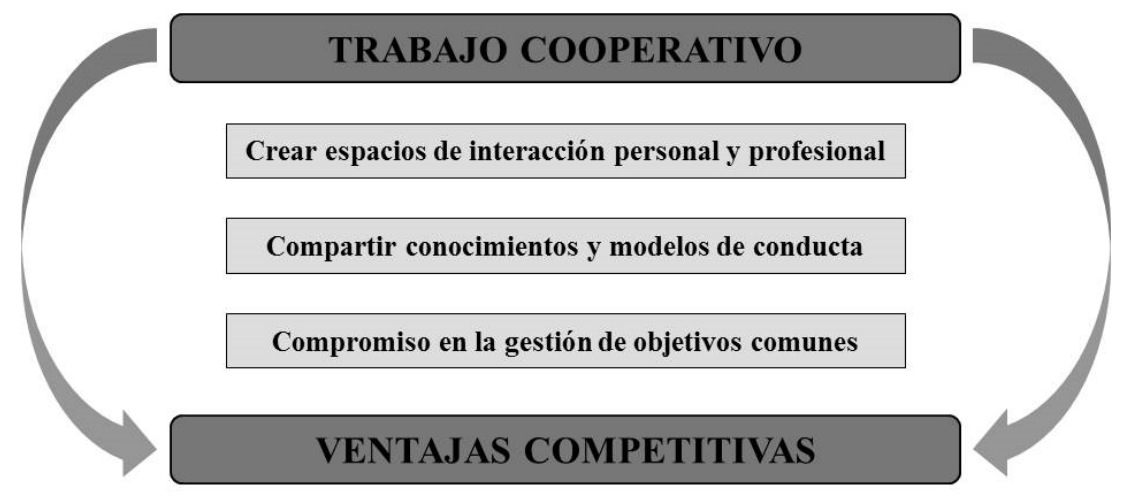

Figura 4. Ventajas del Trabajo Cooperativo en grupo

Una de las potencialidades de esta metodología de trabajo es la heterogeneidad de los componentes del grupo, que lejos de ser un problema se convierte en una ventaja competitiva al crearse un marco de convivencia activa, en el que los participantes se ayudan unos a otros y se responsabilizan del resultado final.

Si bien el planteamiento metodológico se muestra como un sistema perfectamente cerrado, en el que cada miembro del grupo sabe lo que debe hacer y se responsabiliza del resultado final común, existen peligros potenciales que pueden hacer ineficaz esta forma de trabajar. Por una parte, es posible que el grado de implicación de los miembros del grupo no sea el 
mismo ni tampoco el deseado, o que aparezcan rivalidades y problemas de convivencia y sociabilidad por las distintas personalidades y sensibilidades que confluyen (Poveda, 2006). Todo esto es posible y ocurre, pero el trabajo cooperativo implica también a los docentes, que deben posicionarse activamente orientando y dinamizando el grupo de trabajo y mediando en los conflictos que pudieran surgir. Los docentes deben obrar con diligencia buscando un equilibrio entre las exigencias individuales y los compromisos del grupo, analizando previamente las capacidades de cada uno de los miembros que lo forman. (Viles et al, 2010; León et al., 20011; Mesa, 2012)

\subsubsection{Competencias transversales}

El planteamiento de las clases prácticas se ha realizado para que los alumnos adquieran las competencias transversales que relaciona la guía docente. En las clases teóricas también se consideran estas competencias, pero es en las prácticas cuando se interactúa constantemente con los alumnos.

Los dos trabajos prácticos se realizan en grupo por lo que implican capacidad de trabajo en equipo, desarrollo de habilidades en las relaciones personales, capacidad de liderazgo,...

Los alumnos tienen que buscar y analizar información sobre los edificios existentes o las soluciones que planteen en el segundo trabajo, empleando competencias como aprendizaje autónomo, creatividad, análisis y síntesis, razonamiento crítico, capacidad de gestión de la información, actitud positiva frente a las innovaciones sociales y culturales, ...

El primer trabajo se expone en público, de manera aleatoria por uno de los miembros del grupo, evaluándose la exposición, por lo que el alumno debe tener un compromiso con sus compañeros y desarrollar las competencias de comunicación oral y capacidad de comunicación a través de la palabra y la imagen.

En el segundo trabajo desarrolla competencias tales como orientación al cliente (en este caso el docente), capacidad de organización y planificación, resolución de problemas, toma de decisiones, motivación por la calidad, ...

\subsubsection{Desarrollo de los trabajos prácticos}

La asignatura se imparte en un semestre, con trece sesiones prácticas de una hora. El primer trabajo se plantea el día de la presentación y se desarrollará las tres primeras semanas, destinando una o dos clases para la exposición en público de este. Los días de la exposición se explica el contenido del segundo trabajo, que se entregará el último día de las clases prácticas. Para los trabajos prácticos se diseñan grupos de trabajo formados con un máximo de tres alumnos, estableciendo unas normas de organización del trabajo consensuadas entre sus miembros, así como un compromiso de corresponsabilidad en el resultado final. 

sustainability in the Degree in Technical Architecture of the University of Burgos

El objetivo es formar a los alumnos en las técnicas de construcción sostenible utilizadas en edificación y fomentar su interacción personal formando parte de un grupo de trabajo en la consecución de un objetivo común. Con ello se quiere conseguir:

- Contribuir a mejorar el proceso de enseñanza y aprendizaje mediante el trabajo cooperativo.

- Fomentar la creatividad de los alumnos y el interés por nuevas tecnologías sostenibles.

- Socializar al alumno para que perciba y valore las potencialidades del trabajo en grupo y sepa integrarse en los equipos de trabajo de las empresas.

- Adquirir criterios de opinión y de elección para optar por soluciones sostenibles ventajosas en construcción (economía circular).

- Capacidad de contraponer opiniones con argumentos fundamentados en el conocimiento

Como primer trabajo cada grupo elige un edificio que disponga de Certificación de Sostenibilidad o un reconocimiento como tal, para estudiar todos los elementos diferenciadores respecto de un edificio convencional. Se realiza un análisis del diseño arquitectónico sostenible, de los sistemas constructivos, materiales e instalaciones (figura 5), sistemas pasivos, integración en el entorno, empleo de energías renovables...analizando sus puntos fuertes y sus debilidades, tanto en la fase diseño como en el proceso de ejecución y en su mantenimiento, teniendo en cuenta su tipología de uso.

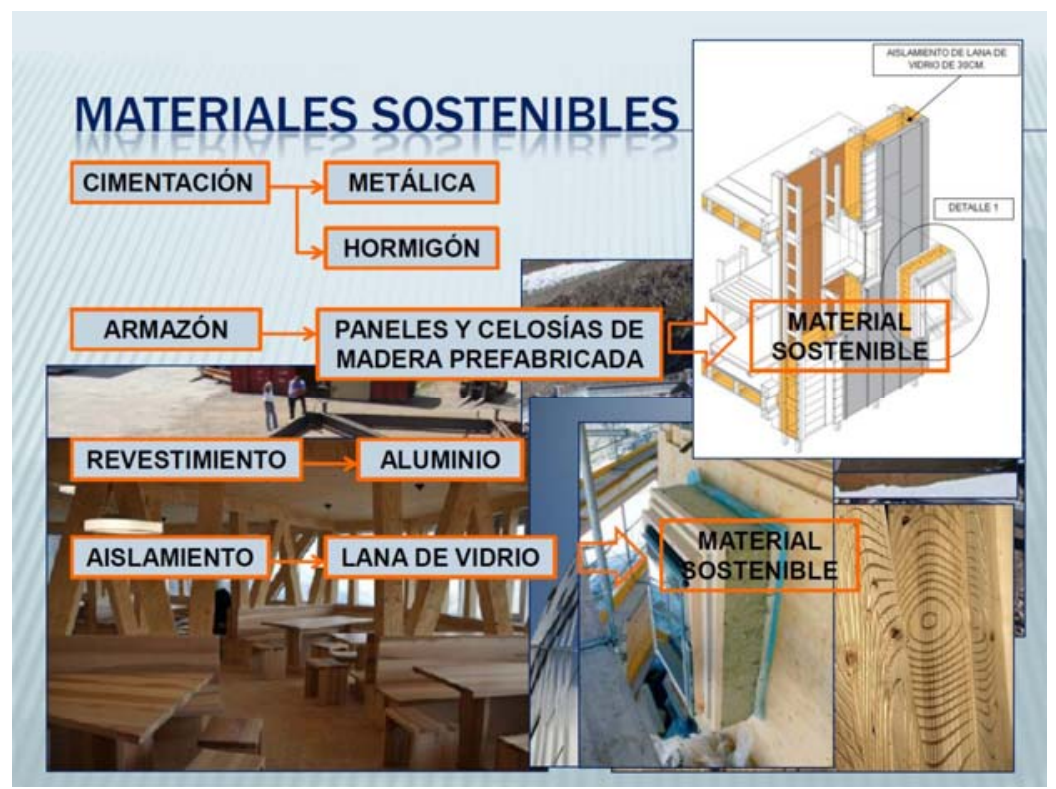

Fig. 5 Construcción Sostenible. Ejemplo de trabajo práctico 1. Estudio de materiales 
Cada grupo de trabajo debe realizar un informe técnico completo que posteriormente se expone en grupo, utilizando técnicas de exposición gráficas basadas en TIC, de este modo se transmite toda la información al resto de la clase. La exposición se realiza de manera similar a un congreso, con tiempos medidos y abriéndose un debate al final de la presentación en el que pueden y deben participar los alumnos.

Como segundo trabajo se entrega a los alumnos un proyecto de edificación convencional para que apliquen los criterios y conocimientos adquiridos en la asignatura y lo transformen en un proyecto de edificación sostenible, figura 6. Para ello pueden modificar todos los elementos constructivos (estructura y cimentación, fachada, cubiertas, particiones, carpinterías, revestimientos...) y las instalaciones, emplear sistemas pasivos (Muro Trombe, tubos canadienses, muro parietodinámico, chimenea solar, etc..) (Neila, 2014, Rodriguez, 2002), y actuar en el entorno que le rodea, ya sea con vegetación o con sistemas de soleamiento. La única limitación es no variar la distribución interior de la edificación, ya que no es objeto de la asignatura.

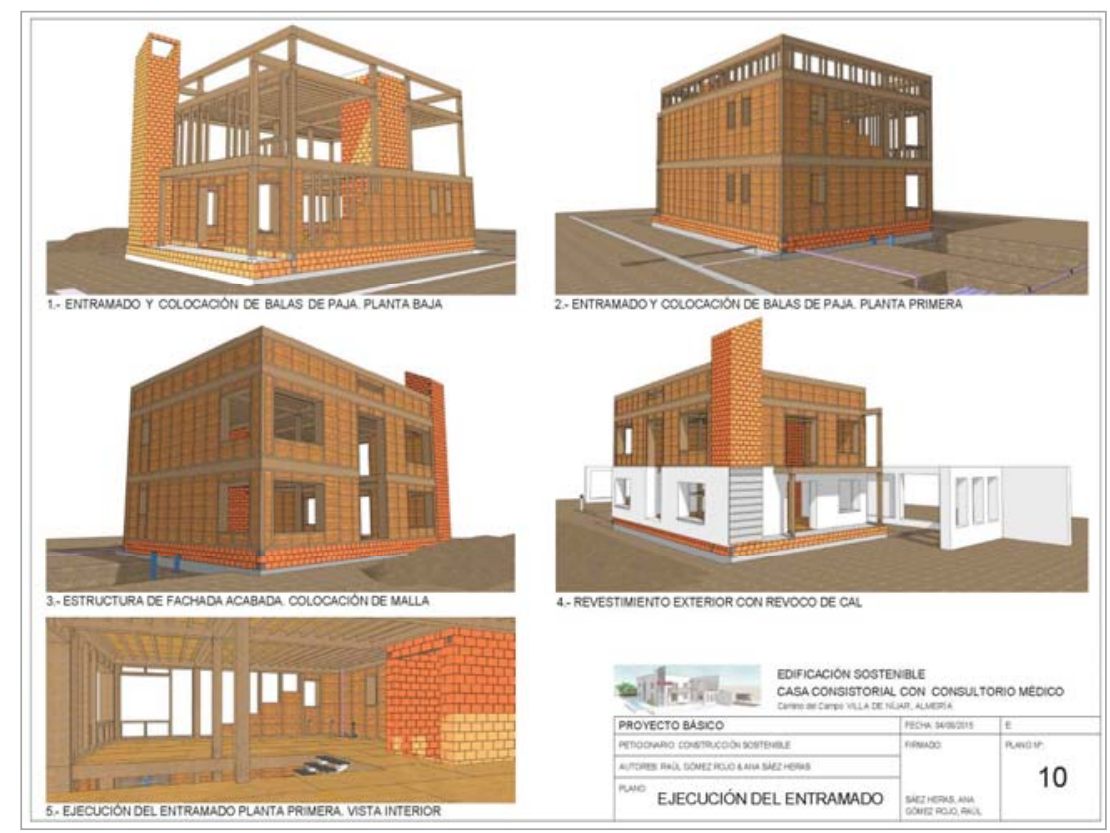

Fig. 6 Construcción Sostenible. Ejemplo de trabajo práctico 2. Desarrollo constructivo

Para que las soluciones propuestas por los alumnos en los trabajos sean más heterogéneas y abarquen diferentes tipologías innovadoras, se plantean diferentes emplazamientos, con climatologías diferenciadas (D’Amico, 2014). Suelen ser cornisa cantábrica, Burgos y Andalucía interior. 
El desarrollo de esta segunda actividad se organiza con los mismos grupos de trabajo establecidos en la primera fase. De igual forma, el protocolo de actuaciones es común para todos los grupos, tomando como referencia los siguientes criterios y planteamientos:

- Análisis del entorno del edificio, estudio del clima, soleamiento, viento y especies vegetales naturales, con el objetivo de poder detectar tanto los beneficios como los factores adversos a la hora de plantear las soluciones arquitectónicas elegidas.

- Análisis del programa de necesidades del edificio y de sus ocupantes, así como de la orientación óptima del mismo, de acuerdo con los factores climatológicos.

- Estudio de las características constructivas y de diseño, de las medidas pasivas y activas, y de las energías renovables del entorno, en función de su uso, y posterior examen crítico mediante el análisis de los indicadores medioambientales.

- Puesta en común y debate sobre las posibles actuaciones para introducir mejoras, de acuerdo con los conocimientos transmitidos en las clases magistrales de la asignatura.

- Debate y puesta en común de las opciones técnicas de aplicación, eligiendo de forma consensuada las propuestas más ventajosas elegidas por cada grupo de trabajo.

- Capacidad de contraponer opiniones con argumentos fundamentados en el conocimiento, con el objetivo de aportar mejoras que sean técnicamente viables.

- Elección de las soluciones constructivas y de las instalaciones de los edificios, indicando las diferentes opciones y tipologías. El documento final del trabajo debe contener una memoria descriptiva razonada, complementada con un estudio gráfico. De igual forma, se establecerá un análisis comparativo entre las diferentes alternativas estudiadas, detallando sus ventajas y defectos.

\section{Conclusiones sobre las estrategias docentes}

La puesta en práctica de esta metodología educativa, basada en el trabajo cooperativo, muestra un alto compromiso de los alumnos y una gran implicación en el desarrollo de los trabajos, así como un elevado interés del alumno por experimentar otras formas de trabajar. La propuesta educativa ayuda a que los alumnos interioricen el trabajo en grupo, ya que experimentan una forma de trabajar real y profesional, tal y como sucede en el ámbito empresarial. A modo de referencia, se pueden aportar las siguientes reflexiones:

- Se percibe que los alumnos asumen el trabajo en grupo como una experiencia profesional, semejante a la que experimentarán en las empresas, una vez concluidos sus estudios.

- La exposición de los trabajos permite desarrollar otras competencias personales, como la capacidad de comunicar, de explicar un proyecto, de fundamentar y defender con argumentos las soluciones adoptadas y de convencer a la audiencia. 
- Son capaces de comparar su trabajo con el del resto de compañeros, lo que motiva su interés por mejorar y conseguir la excelencia del grupo.

- El alumno valora y respeta el trabajo del resto de compañeros, aceptando la existencia de otros puntos de vista y opiniones, lo que contribuye a enriquecer el nivel colectivo de los trabajos.

\section{Referencias}

D'AMICO, F. C. (2014). Arquitectura bioclimática, conceptos básicos y panorama actual. Boletín CF+ S, (14). http://habitat.aq.upm.es/boletin/n14/afcel.html

DE LA TORRE, S. ; VIOLANt, V. (2001). Estrategias creativas en la enseñanza universitaria. Creatividad y sociedad, 3, 10-35.

EDWARDS, B. (2008) Guía básica de la sostenibilidad, Editorial Gustavo Gili, Barcelona.

HERNANDEZ PEZZI, C. (2007) Un Vitruvio ecológico: Principios y práctica del proyecto arquitectónico sostenible, Editorial Gustavo Gili, Barcelona.

HEYWOOD, H. (2017) 101 reglas básicas para edificios y ciudades sostenibles , Gustavo Gili, Barcelona.

LEON, B., Felipe, E., Iglesias, D. y Latas, C. (2011). El aprendizaje cooperativo en la formación inicial del profesorado. Revista de Educación, 354.

MARIN, V. ; NEGRE, F. ; PEREZ, A. (2014). Entornos y redes personales de aprendizaje (PLE-PLN) para el aprendizaje colaborativo. Comunicar, 21(42).

MESA, L. M. (2011). El trabajo colaborativo del profesorado como oportunidad formativa. Los docentes, conciencia educativa de la sociedad, 69.

NEILA, J. (2014). Arquitectura bioclimática en un entorno sostenible: buenas prácticas edificatorias. Boletín CF+ S, (14). http://habitat.aq.upm.es/boletin/n14/afcel.html

POVEDA SERRA, P. (2006). Implicaciones del aprendizaje de tipo cooperativo en las relaciones interpersonales y en el rendimiento académico. Universidad de Alicante.

REYES, C.; BARAHONA, E.; PIRILLO, C. (2007) Arquitectura sostenible, Editorial Pensil, Valencia.

RODRIGUEZ, M. (2002) Introducción a la arquitectura bioclimática, Limusa, México,

SAIZ, M. S. I. ; GOMEZ, G. R. (2011). Aprendizaje autónomo y trabajo en equipo: reflexiones desde la competencia percibida por los estudiantes universitarios. Revista Electrónica Interuniversitaria de Formación del Profesorado (REIFOP), 73

VILES, E., Jaca, C., Campos, J., Serrano, N., \& Santos, J. (2012). Evaluación de la competencia de trabajo en equipo en los grados de ingeniería. Dirección y Organización, (46), 67-75. 